\title{
Updated Classification Schemes for Fibro-Osseous Lesions of the Oral \& Maxillofacial Region: A Review
}

\author{
Dr.Karan Rajpal, Dr.Raghav Agarwal, Dr.Richie Chhabra, \\ Dr.Maumita Bhattacharya
}

\begin{abstract}
Fibro-Osseous lesions [FOL] are a group of lesions which are known to affect the jaws and the craniofacial bones which is regarded as very confusing area in diagnostic pathology. The term refers to a diverse process in which the normal architecture of bone is replaced by fibrous tissue containing varying amount of foci of mineralization. Various classification systems have been put forward by various authors by extensive research methodologies. The purpose of this present article is to propose the various classification systems given by various authors which will enable us to adopt a uniform terminology and improve communications between clinicians, pathologist and surgeons.

Key Words: Fibro-Osseous lesions, WHO, Classification
\end{abstract}

\section{Introduction}

Fibro-Osseous lesions [FOL] are a group of lesions which are known to affect the jaws and the craniofacial bones which is regarded as very confusing area in diagnostic pathology. The term refers to a diverse process in which the normal architecture of bone is replaced by fibrous tissue containing varying amount of foci of mineralization. ${ }^{[1]}$ These group of lesions are known to encompass common characteristics that include common clinical, radiographic and microscopic features. Most of these lesions are of unknown aetiology, while some lesions are believed to be neoplastic while others are related to metabolic disturbances and are also believed to cause considerable diagnostic challenges. ${ }^{[2]}$ While some of the FOL are believed to be true neoplasms having a considerable potential for growth if not fully excised, the peculiar anatomic location of these craniofacial lesions can result in conditions like encephalitis and sometime meningitis which are considered fatal. ${ }^{[3]}$

Histologically, these group of FOL are characterised by the replacement of normal bone by fibrous connective tissue matrix. The fibrous tissue displays variable features like varying degree of mineralization in the form of woven bone or cementum like basophilic structures which are indistinguishable from cementicles. ${ }^{[4]}$

Classification Schemes of Fibro-Osseous Lesions

The various Classifications systems proposed by authors are enumerated as below.

- Charles Waldron Classification Of The Fibro-Osseous Lesions Of The Jaws (1985)

- Working Classification Of Fibro-Osseous Lesions By Mico M. Malek (1987)

- $\quad$ Peiter J. Slootweg \& Hellmuth Muller (1990)

- WHO Classification (1992)

- Waldron Modified Classification Of Fibro-Osseous Lesions Of Jaws (1993)

- Brannon \& Fowler Classification (2001)

- WHO Classification Of Fibro-Osseous Lesions Of Jaws (2005)

- $\quad$ Paul M. Speight \& Roman Carlos Classification (2006)

- Eversole Classification (2008)

Charles Waldron Classification Of The Fibro-Osseous Lesions Of The Jaws (1985) ${ }^{[5]}$

1. Fibrous Dysplasia

a. Monostotic

b. Polyostotic

2. Fibro-Osseous (Cemental) Lesions Presumably Arising In The Periodontal Ligament

a. Periapical Cemental Dysplasia

b. Localized Fibro-Osseous-Cemental Lesions (Probably Reactive In Nature)

c. Florid Cement-Osseous Dysplasia (Gigantiform Cementoma)

d. Ossifying \& Cemenifying Fibroma

3. Fibro-Osseous Neoplasms Of Uncertain Or Detectable Relationship To Those Arising In The Periodontal Ligament (Category II) 
a. Cemetoblastoma, Osteoblastoma \& Osteoid Osteoma

b. Juvenile Active Ossifying Fibroma \& Other So Called Aggressive, Active Ossifying /Cementifying Fibromas.

Working Classification Of Fibro-Osseous Lesions By Mico M. Malek (1987) ${ }^{[6]}$

In 1987 from the viewpoint of diagnostic pathologist, a working classification of fibro-osseous lesions was given by Mico M. Malek which is as follows

\section{Developmental Disorders}

A. Fibrous Cortical Defects (Non Ossifying Fibroma)

B. Fibrous Dysplasia

2. Reactive Reparative Lesions
A. Traumatic Periosteitis
B. Periosteitis Ossificans
C. Osseous Keloid
D. Periapical Cemental Dysplasia \& Florid Cemento-Osseous Dysplasia
E. Sclerosing Osteomyelitis (Focal \& Diffuse Type)
F. Osteitis Deformans

3. Fibromatosis

4. Neoplasms

A. Desmoplastic Fibroma (Intraosseous Fibromatosis)

A. Tooth Bearing Areas Only

i. Cementoblastoma

ii. Periodontoma

1. Central

2. Peripheral

B. All Cranio-Facial Bones (Including Tooth Bearing Areas)

i. Osteoma

1. Trabecular

2. Compact

ii. Osteoid Osteoma

iii. Psammous Desmo-Osteoblastoma

iv. Trabecular Desmo-Osteoblastoma

Peiter J. Slootweg \& Hellmuth Muller (1990) ${ }^{[7]}$

In 1990 Peiter. J. Slootweg \& Hellmuth Muller gave a classification that laid emphasis primarily on the histopathological features, and they underscore that this classification requires inclusion of adjacent normal bone to make diagnosis. However in the absence of this, the clinical \& radiological features have to be taken in to consideration.

Group I: Fibrous Dysplasia

Group II: Juvenile Ossifying Fibroma

Group III: Ossifying Fibroma

Group IV: Periapical Cemental Dysplasia \& Florid Osseous Dysplasia

\section{WHO Classification (1992) ${ }^{[8]}$}

But the identification of identical cementum like tissues in lesions in extra-gnathic sites suggested that this tissues may be a merely normal variant of bone, and that dental cementum itself is a specialized form of "bundle-bone". Therefore, in the second edition of the who's classification in 1992, three of the "cemental" lesion were transferred to the "neoplasm and other tumors related to bone "group, leaving the benign cementoblastoma as the sole true neoplasm of dental cementum. ${ }^{[9]}$

This second edition of the WHO Histological Typing of odontogenic tumors in 1992 recognized them as the group of cement-osseous dysplasia which included "florid cement-osseous dysplasia" that form with "Periapical cemental dysplasia" \& "other cemento-osseous dysplasia"

\section{Osteogenic Neoplasms}

a. A.Cemento-Ossifying Fibroma (Cementifying Fibroma, Ossifying Fibroma)

\section{Non-Neoplastic Bone Lesions}

a. Fiberous Dysplasia Of Jaws

b. Cemento-Osseous Dysplasia

I. Periapical Cemental Dysplasia (Periapical Fiberous Dysplasia), 
II. Florid Cemento-Osseous Dysplasia (Gigantiform Cementoma, Familial Multiple Cementomas)

III. Other Cemento-Osseous Dysplasia

c. Cherubism (Familial Multilocular Cystic Disease Of The Jaws)

d. Central Giant Cell Granuloma

e. Aneurismal Bone Cyst

f. Solitary Bone Cyst (Traumatic, Simple, Hemorrhagic Bone Cyst)

\section{Waldron Modified Classification Of Fibro-Osseous Lesions Of Jaws (1993) ${ }^{[10]}$}

Later on, to overcome the demerits of his own classification, Waldron reviewed the subject of benign fibro-osseous lesions of jaws (BFOL) in 1993 and suggested a modification of his earlier classification.

1. Fibrous Dysplasia

2. Cement-Osseous Dysplasia

a. Periapical Cement-Osseous Dysplasia

b. Focal Cement-Osseous Dysplasia

c. Florid Cement-Osseous Dysplasia

\section{Fibro-Osseous Neoplasm}

a. Cementifying Fibroma, Ossifying Fibroma, Cement-Ossifying Fibroma

Brannon \& Fowler Classification (2001) ${ }^{[11]}$

In 2001, Brannon \& Fowler gave another classification which was quite different from that of Waldron \& WHO classification. this was done to include more number of lesions which were also showing features like FOL:

1. Osseous Dysplasia (OD) (Reactive)

a. Nonhereditary

i. Periapical

ii. Focal

iii. Florid

b. Hereditary (Developmental)

i. Familial Gigantiform Cementoma

2. Fibro-Osseous Neoplasm

a. Ossifying Fibroma (OF)

b. "Juvenile", "Active" or "Aggresive" Varients of OF

3. Fibrous Dysplasia
a. Polyostotic FD
b. Monostotic FD
c. Craniofacial FD

4. Giant Cell Lesions
a. Central Giant Cell Granuloma
b. Aneurismal Bone Cyst
c. Cherubism

5. Miscellaneous Benign Fibro-Osseous Lesions
a. Cementoblastoma
b. Tori/Exostoses
c. Osteoma

\section{WHO Classification Of Fibro-Osseous Lesions Of Jaws (2005) ${ }^{[8]}$}

In the latest WHO's classification of odontogenic tumors in 2005, COD has been therefore called osseous dysplasias (Barnes Et Al.). because the discussions during these last decades about whether cementumlike tissues is present, it has been decided to give up the term of "cement". The core of this classification is the concept of a spectrum of clinicopathological entities in which the diagnosis can only be made on the basis of a full consideration of clinical, histological and radiological features. ${ }^{14}$

1) Ossifying Fibroma (OF)

2) Fiberous Dysplasia

3) Osseous Dysplasia

a. Periapical Osseous Dysplasia

b. Focal Osseous Dysplasia

c. Florid Osseous Dysplasia 
4) Central Giant Cell Granuloma

5) Cherubism

6) Aneurismal Bone Cyst

7) Solitary Bone Cyst

Paul M. Speight \& Roman Carlos Classification (2006) ${ }^{[1]}$

In 2006, Paul M. Speight \& Roman Carlos gave a classification based on new WHO classification \& also from Waldron, Slootweg, Brannon and Fowler and El-Mofty. A number of workers have tried to clarify the classification of these lesions and although that may not have agreed on an exact terminology, a concept has emerged which has culminated in the latest WHO classification. Although the terminology is still problematic, this new classification concentrated on the histopathological features that may guide the working surgical pathologist towards a diagnosis. ${ }^{11}$

1. Fibrous Dysplasia

a. Monostotic FD

b. Polyostotic FD

c. Craniofacial FD

2. Osseous Dysplasia
a. Periapical Osseous Dysplasia
b. Focal Osseous Dysplasia
c. Florid Osseous Dysplasia
d. Familial Gigantiform Cementoma

\section{Ossifying Fibroma}

a. Conventional Ossifying Fibroma

b. Juvenile Trabecular Ossifying Fibroma

c. Juvenile Psammomatoid Ossifying Fibroma

\section{Eversole 2008 Classification ${ }^{[12]}$}

Further, a much more comprehensive classification has been suggested by Eversole et al in 2008 and this suggests that the classification of these disease is likely to evolve still further. this classification includes neoplasm, developmental dysplastic lesions and inflammatory/reactive processes. The basis of this classification is that definitive diagnosis can rarely be rendered on the basis of histopathological features alone rather; procurement of a final diagnosis is usually dependent upon assessment of microscopic, clinical and imaging features together. ${ }^{13}$

Classification of Benign Fibro-Osseous Lesions of the Craniofacial Complex:

\section{Bone dysplasias}

a. Fibrous dyspla

i. Monostotic

ii. Polyostotic

iii. Polyostotic with endocrinopathy (McCune-Albright) iv Osteofibrous dysplasiaa

b. Osteitis deformans

c. Pagetoid heritable bone dysplasias of childhood

d. Segmental odontomaxillary dysplasia

2. Cemento-osseous dysplasias

a. Focal cemento-osseous dysplasia

b. Florid cemento-osseous dysplasia

3.Inflammatory/reactive processes

a. Focal sclerosing osteomyelitis

b. Diffuse sclerosing osteomyelitis

c. Proliferative periostitis

4. Metabolic Disease: hyperparathyroidism

5. Neoplastic lesions (Ossifying fibromas)

a. Ossifying fibroma

b. Hyperparathyroidism jaw lesion syndrome

c. Juvenile ossifying fibroma

i. Trabecular type

ii. Psammomatoid type 


\section{d. Gigantiform cementomas}

\section{Discussion}

Fibro- Osseous Lesions of the jaw have been under frequent renaming and reclassification due to its varied features. ${ }^{[9]}$ The similarity between all the FOL of the jaw is the replacement of the normal bone with fibrous connective tissue with interspersed mineralized product, that includes osteoid, mature bone or presence of cementum like calcifications. The major challenge faced by the author are further sub classifying the lesions. ${ }^{[4]}$ There has always been constant disagreement regarding the

Nomenclature of benign fibro-osseous lesions, due in part to the peculiar pathological patterns of stroma and bone in these group of lesions and even similar or identical microscopic features can be in common amongst two or more different lesions. ${ }^{[12]}$

Over the years various classification models have been put forward of which the Waldron's classification system has gained considerable recognition over the years. The classification system of Waldron has suggested that the FOL originate from the periodontal ligament which contains multipotent cells which are known to differentiate into fibrous tissue cells, cementum and bone.

Eversole has classified these Fibro-Osseous lesions according to pathogenetic mechanisms yet the most suitable approach for diagnosis is to consider FOL in the context of combined clinical, radiologic and pathologic characteristics. It is unquestionable that a definitive diagnosis should be given only on the basis of microscopic features alone, and other variables like age sex and race should be considered during the formulation of a final diagnosis. In the forthcoming times new genetic and molecular finding of the FOL will necessitate further modification and subsequent changes in the classification systems. Hence a new and revised version of the classification will always be required.

References

[1]. Speight P.M, Carlos R. Maxillofacial fibro-osseous lesions .Current Diagnostic Pathology .2006;12:1-10

[2]. Bahl S, Sandhu S , Gupta M. BENIGN FIBRO-OSSEOUS LESIONS OF JAWS- A REVIEW. INTERNATIONAL DENTAL JOURNAL OF STUDENT'S RESEARCH. 2012;1(2):56-68

[3]. Hall G. Fibro-Osseous lesions of Head and Neck.Diagnostic Histoplathology .2012;18(4):149-158

[4]. Alawi F. Benign fibro-osseous diseases of the maxillofacial bones. A review and differential diagnosis. Am $J$ Clin Pathol. 2002 Dec;118 Suppl:S50-70.

[5]. Waldron CA. Fibro-osseous lesions of the jaws. J Oral Maxillofac Surg. 1985 Apr;43(4):249-62.

[6]. Makek MS. So called "fibro-osseous lesions" of tumorous origin. Biology confronts terminology. J Craniomaxillofac Surg. 1987 Jun;15(3):154-67

[7]. Slootweg PJ, Müller H. Differential diagnosis of fibro-osseous jaw lesions. A histological investigation on 30 cases. J Craniomaxillofac Surg. 1990 Jul;18(5):210-4

[8]. Benjellou L , Harti KE, Wady WE.Florid Osseous dyplasia: Report of two cases and review of literature. Int J Odontostomat.2011;5(3):257-266

[9]. MacDonald-Jankowski DS. Fibro-osseous lesions of the face and jaws. Clin Radiol. 2004 Jan;59(1):11-25

[10]. Akcam T , Ayberk H . Synchronus Ossifying Fibroma: A Review.Oral Surg Oral med Pathol Oral radiol Endod.2012;114(5):120125

[11]. Brannon RB, Fowler CB. Benign fibro-osseous lesions: a review of current concepts. Adv Anat Pathol. 2001 May;8(3):126-43

[12]. Eversole R, Su L, ElMofty S. Benign fibro-osseous lesions of the craniofacial complex. A review. Head Neck Pathol. 2008 Sep;2(3):177-202 